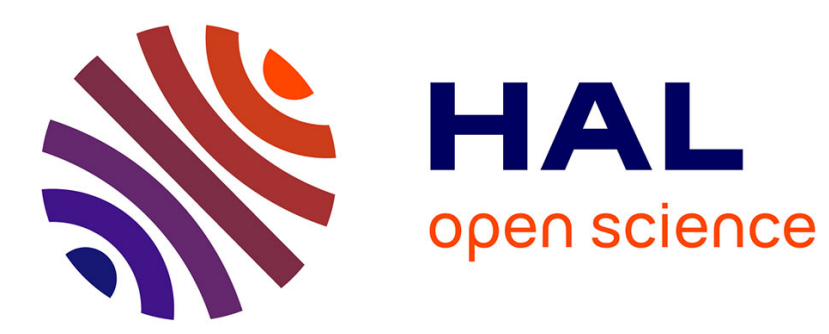

\title{
Exponential stabilization of a class of flexible microgrippers using dynamic boundary port Hamiltonian control.
}

\author{
Héctor Ramirez Estay, Yann Le Gorrec, Hans Zwart
}

\section{- To cite this version:}

Héctor Ramirez Estay, Yann Le Gorrec, Hans Zwart. Exponential stabilization of a class of flexible microgrippers using dynamic boundary port Hamiltonian control.. 52nd IEEE Conference on Decision and Control, CDC'13., Jan 2013, Italy. pp.1-6. hal-00910963

\section{HAL Id: hal-00910963 https://hal.science/hal-00910963}

Submitted on 28 Nov 2013

HAL is a multi-disciplinary open access archive for the deposit and dissemination of scientific research documents, whether they are published or not. The documents may come from teaching and research institutions in France or abroad, or from public or private research centers.
L'archive ouverte pluridisciplinaire HAL, est destinée au dépôt et à la diffusion de documents scientifiques de niveau recherche, publiés ou non, émanant des établissements d'enseignement et de recherche français ou étrangers, des laboratoires publics ou privés. 


\title{
Exponential stabilization of a class of flexible microgrippers using dynamic boundary port Hamiltonian control.
}

\author{
Hector Ramirez, Yann Le Gorrec and Hans Zwart
}

\begin{abstract}
This paper deals with the control of a class of simplified models for flexible micro-grippers for DNA manipulation. The overall system is first modelled as a boundary controlled port Hamiltonian system made up as the interconnection of an infinite dimensional system (modelled as an undamped Timoshenko beam) representing the flexible arm of the gripper with two finite dimensional systems representing the DNA bundle and the suspension/actuator mechanism. The base of the arm is clamped on the suspension mechanism leading to under actuated system. The controller considered under strict dissipative port Hamiltonian format uses the velocity of the base of the tweezers arm as input and generates a force as output. The exponential stability of the closed loop system is derived by checking simple conditions on both the infinite and finite dimensional system.
\end{abstract}

\section{INTRODUCTION}

The manipulation of biological molecules by using micromechanical and optical devices such as: magnetic tweezers [1], [2], optical tweezers [3], AFM cantilevers [4] and microfibers [5], [6], are nowadays possible thanks to impressive technological progresses of the last years. The molecules manipulation is of particular interest for the characterization of basic properties of biological molecules such as DNA molecules [7]. Silicon nanotweezers, (Figure 1). are used for

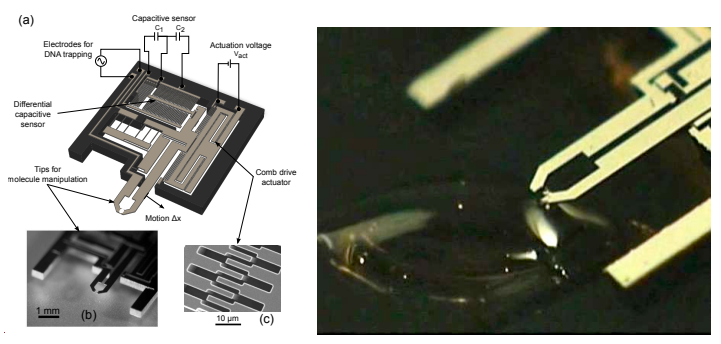

Fig. 1. Silicon nanotweezers

this purpose: the principle is to trap DNA bundle between two arm tips using dielectrophoresis and then to characterize the DNA mechanical properties by using electrostatic actuation. The actuator is also used for monitoring enzymatic reactions on the DNA. The nanotweezers are so sensitive to

The contribution of Y. Le Gorrec and H. Ramirez has been done within the context of the NRA project HAMECMOPSYS with reference code ANR-11-BS03-0002. Further information is available at http://www.hamecmopsys.ens $2 \mathrm{~m} . \mathrm{fr} /$.

H. Zwart is with the Department of Applied Mathematics, Faculty of Electrical Engineering, Mathematics and Computer Science, Univer sity of Twente, P.O. Box 217, 7500 AE Enschede, The Netherlands h.j.zwart@math.utwente.nl

Y. Le Gorrec and H. Ramirez are with FEMTO-ST AS2M, 24 rue Alain Savary, 25000 Besançon, France Yann. Le. Gorrec@ens2m. fr the stiffness variation of the DNA bundle that they become flexible and as consequence, current microfabrication processes tend to reduce the thickness of the beams in order to improve the sensitivity of the actuator. This leads to control problems that may be formulated in terms of partial differential equations (PDEs).

The framework of infinite dimensional port Hamiltonian systems has proven to be a powerful tool for the modelling and control of systems described by PDEs. Indeed, the definition of Boundary Controlled port Hamiltonian Systems (BCpHS) [8], [9], [10], [11], [12], have made it possible to develop constructive results analysis of well-posedness and stability of solutions.

In [13] it has been shown that the interconnection of a fully actuated BCpHS with a linear finite dimensional controller renders the closed-loop system exponentially stable provided that the finite dimensional system is input strictly passive and exponentially stable. This result permits to elegantly, and quite easily, prove the exponential stability for a large class of linear controllers, in particular those arising from energy shaping methods using Casimir functions [14], [15].

In this paper we use BCpHS to model and prove the exponential stability of a class of simplified models of underactuated silicon nanotweezer for DNA manipulation. More specifically the port Hamiltonian framework is used to construct a model of the manipulation process by interconnecting the different sub-systems: The infinite dimensional part consisting on the flexible arm of the gripper, modelled as an undamped Timoshenko beam; and the finite dimensional parts consisting on the DNA-bundle and the suspension/actuator mechanism. Preliminary results on a simplified version of the model have been presented in [16], [17]. The stabilization of the process is performed by using a strict dissipative port Hamiltonian controller acting on the base of the tweezer through the transversal force only, the arm of the tweezers being clamped. We show that even when consider the extreme case of an undamped flexible arm, the closed-loop system is exponentially stable provided that the boundary controller is input strictly passive. The practical derivation of such controller (by using immersion/reduction method through Casimir invariants for example) is not detailed here. We focus in this paper on the analysis of the exponential stability of the underactuated closed loop system by checking simple conditions on both the infinite and finite dimensional systems and adapting the general result proposed in [13].

The paper is organized as follows: The first section presents to port Hamiltonian models of the tweezer, the 
DNA and the suspension system as well as the model of the whole system after interconnection. The second section is devoted to exponential stability analysis. The paper ends with a conclusion and some perspectives.

\section{Port Hamiltonian modelling of the FleXible MICROGRIPPER}

In this paper we consider the simplified model of a flexible silicon based microgripper (Figure 2) used for DNA manipulation [18]. We assume that the tweezer is made up of

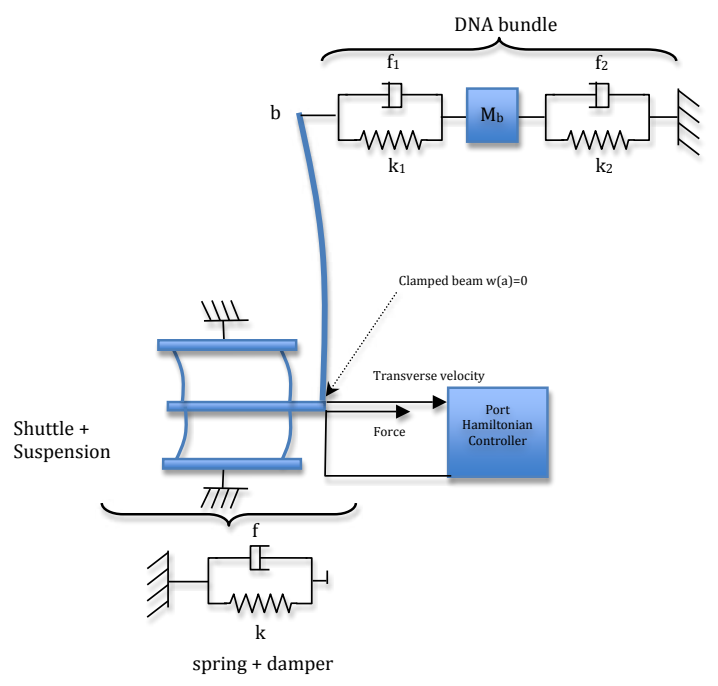

Fig. 2. DNA manipulation through port Hamiltonian control

largely flexible arm, that can be considered as a flexible beam with large deflection clamped to a transverse suspension system. The trapped DNA bundle is approximated by a spring/damper-mass-spring/damper system attached at the tip of the tweezer. The arm is actuated by using electrostatic forces generated by a comb drive actuator (not represented in Figure 2). Contrary to the model proposed in [16] (in which both force and torque at point $a$ are used as control variables) we consider here the dynamics of the suspension system (the spring stiffness and damping coefficients are in a first instance considered constant). We assume that it is only possible to measure the transversal velocity at point $a$. The

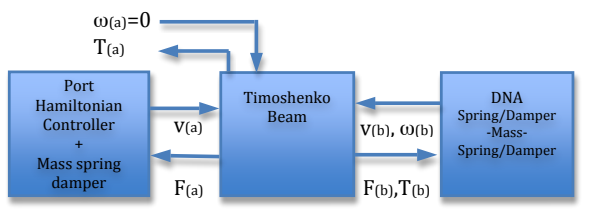

Fig. 3. Interconnection and control strategy

whole system can be divided into three subsystems (Figure 3), namely the flexible arm, the DNA-bundle at the tip of the gripper and the actuated shuttle. Since we assume that the the flexible arm admits large deflections, we model it as a Timoshenko beam (infinite dimensional system). Furthermore, we consider the extreme case of an undamped Timoshenko beam, i.e, without dissipation. The choice of an undamped beam is motivated by control considerations. It is well known that dissipation adds robustness to a passive control scheme. Hence, by considering an undamped Timoshenko beam we are situating the control problem in a worst case scenario. We will show that the control is exponentially stable even when the natural damping is not considered.

The DNA-bundle is modelled as a finite dimensional mechanical system. The subsystems are interconnected through their boundary power conjugated port variables. Let's note that only a part of the boundary port variables are used for the interconnection with the suspension system. The other part is considered equal to zero to account for the clamping of the beam. In Figure 3 the interconnection boundary port variables as well as the causality (depicted with arrows) are given. It is worth noting that even if the input of the beam at point a is a velocity (resp. angular velocity) (that is used for the interconnexion), the output of the port Hamiltonian controller is the force applied to the shuttle.

\section{A. The Timoshenko beam}

The damped and undamped Timoshenko beam has been widely studied as a distributed parameter port Hamiltonian system [14] and as BCS [9] and the exponential stability of the system has been proved for static boundary feedback [10], [11]. The BCS is defined as

$$
\begin{aligned}
\frac{\partial}{\partial t}\left[\begin{array}{l}
x_{1} \\
x_{2} \\
x_{3} \\
x_{4}
\end{array}\right]=\underbrace{\left[\begin{array}{llll}
0 & 1 & 0 & 0 \\
1 & 0 & 0 & 0 \\
0 & 0 & 0 & 1 \\
0 & 0 & 1 & 0
\end{array}\right]}_{P_{1}} \frac{\partial}{\partial z}\left[\begin{array}{c}
K x_{1} \\
\frac{1}{\rho} x_{2} \\
E I x_{3} \\
\frac{1}{I_{\rho}} x_{4}
\end{array}\right] \\
+\underbrace{\left[\begin{array}{cccc}
0 & 0 & 0 & -1 \\
0 & 0 & 0 & 0 \\
0 & 0 & 0 & 0 \\
1 & 0 & 0 & 0
\end{array}\right]}_{P_{0}}\left[\begin{array}{c}
K x_{1} \\
\frac{1}{\rho} x_{2} \\
E I x_{3} \\
\frac{1}{I_{\rho}} x_{4}
\end{array}\right]
\end{aligned}
$$

where the following state (energy) variables have been defined: $x_{1}=\frac{\partial w}{\partial z}(z, t)-\phi(z, t)$ the shear displacement, $x_{2}=\rho(z) \frac{\partial w}{\partial t}(z, t)$ the transverse momentum distribution, $x_{3}=\frac{\partial \phi}{\partial z}(z, t)$ the angular displacement, and $x_{4}=I_{\rho} \frac{\partial \phi}{\partial t}(z, t)$ the angular momentum distribution, for $z \in(a, b), t \geq 0$, where $w(t, z)$ is the transverse displacement of the beam and $\phi(t, z)$ is the rotation angle of a filament of the beam. The coefficients $\rho(z), I_{\rho}(z), E(z), I(z)$ and $K(z)$ are the mass per unit length, the rotary moment of inertia of a cross section, Youngs modulus of elasticity, the moment of inertia of a cross section, and the shear modulus respectively. The matrices $P_{1}$ and $P_{0}$ defines the skew-symmetric differential operator of order 1 acting on the state space $X=L_{2}\left(a, b, \mathbb{R}^{4}\right), \mathcal{J}=P_{1} \frac{\partial}{\partial z}+P_{0}$. The energy of the beam 
is expressed in terms of the energy variables,

$$
\begin{aligned}
E & =\frac{1}{2} \int_{a}^{b}\left(K x_{1}^{2}+\frac{1}{\rho} x_{2}^{2}+E I x_{3}^{2}+\frac{1}{I_{\rho}} x_{4}^{2}\right) d z \\
& =\frac{1}{2} \int_{a}^{b} x(z)^{\top}(\mathcal{L} x)(z) d z=\frac{1}{2}\|x\|_{\mathcal{L}}^{2}
\end{aligned}
$$

where $\mathcal{L}=\operatorname{diag}\left(K, \frac{1}{\rho}, E I, \frac{1}{I_{\rho}}\right)$. The boundary port variables are obtained by using integration by parts and factorization in order to define an extended Dirac structure including the boundary [9]. They also can be directly parametrized from $P_{1}$ [9], [10] leading to:

$$
\left[\begin{array}{c}
f_{\partial, \mathcal{L} x} \\
e_{\partial, \mathcal{L} x}
\end{array}\right]=\left[\begin{array}{c}
\left(\rho^{-1} x_{2}\right)(b)-\left(\rho^{-1} x_{2}\right)(a) \\
\left(K x_{1}\right)(b)-\left(K x_{1}\right)(a) \\
\left(I_{\rho}^{-1} x_{4}\right)(b)-\left(I_{\rho}^{-1} x_{4}\right)(a) \\
\left(E I x_{3}\right)(b)-\left(E I x_{3}\right)(a) \\
\left(\rho^{-1} x_{2}\right)(b)+\left(\rho^{-1} x_{2}\right)(a) \\
\left(K x_{1}\right)(b)+\left(K x_{1}\right)(a) \\
\left(I_{\rho}^{-1} x_{4}\right)(b)+\left(I_{\rho}^{-1} x_{4}\right)(a) \\
\left(E I x_{3}\right)(b)+\left(E I x_{3}\right)(a)
\end{array}\right] .
$$

The control objective is to control the translational and angular position of the DNA-bundle. From the interconnection structure, the input and output of the beam are selected as follows:

$$
\begin{aligned}
& u=\left[\begin{array}{llll}
v(b) & \omega(b) & -v(a) & -\omega(a)
\end{array}\right], \\
& y=\left[\begin{array}{llll}
F(b) & T(b) & F(a) & T(a)
\end{array}\right],
\end{aligned}
$$

which is achieved by defining

$$
u=W\left[\begin{array}{l}
f_{\partial, \mathcal{L} x} \\
e_{\partial, \mathcal{L} x}
\end{array}\right], \quad y=\tilde{W}\left[\begin{array}{l}
f_{\partial, \mathcal{L} x} \\
e_{\partial, \mathcal{L} x}
\end{array}\right],
$$

where

$$
\begin{aligned}
W & =\left[\begin{array}{cccccccc}
1 & 0 & 0 & 0 & 0 & 1 & 0 & 0 \\
0 & 0 & 1 & 0 & 0 & 0 & 0 & 1 \\
1 & 0 & 0 & 0 & 0 & -1 & 0 & 0 \\
0 & 0 & 1 & 0 & 0 & 0 & 0 & -1
\end{array}\right], \\
\tilde{W} & =\left[\begin{array}{cccccccc}
0 & 1 & 0 & 0 & 1 & 0 & 0 & 0 \\
0 & 0 & 0 & 1 & 0 & 0 & 1 & 0 \\
0 & -1 & 0 & 0 & 1 & 0 & 0 & 0 \\
0 & 0 & 0 & -1 & 0 & 0 & 1 & 0
\end{array}\right] .
\end{aligned}
$$

It can by shown that with this choice of input and output the system (1) defines a an abstract boundary control system. Furthermore $\mathcal{A} x=P_{1}(\partial / \partial z)(\mathcal{L} x)+P_{0} \mathcal{L} x$ with domain

$$
D(\mathcal{A})=\left\{\mathcal{L} x \in H^{1}\left(a, b ; \mathbb{R}^{n}\right) \mid\left[\begin{array}{c}
f_{\partial, \mathcal{L} x}(t) \\
e_{\partial, \mathcal{L} x}(t)
\end{array}\right] \in \operatorname{ker} W\right\}
$$

generates a contraction semigroup on $X$ and the energy balance equation is defined as:

$$
\frac{d E}{d t}=u^{T} y
$$

In what follows the beam is clamped at point a. We then split the input/ouput in:

$$
u=\left[u_{1}, u_{2}\right], y=\left[y_{1}, y_{2}\right]
$$

with

$$
\begin{aligned}
& u_{1}=\left[\begin{array}{lll}
v(b) & \omega(b) & -v(a)
\end{array}\right], u_{2}=[\omega(a)], \\
& y_{1}=\left[\begin{array}{lll}
F(b) & T(b) & F(a)
\end{array}\right], y_{2}=[T(a)],
\end{aligned}
$$

Furthermore

$$
\left[\begin{array}{l}
u_{1} \\
u_{2} \\
y_{1} \\
y_{2}
\end{array}\right]=\frac{1}{\sqrt{2}}\left[\begin{array}{l}
W \\
\widetilde{W}
\end{array}\right]\left[\begin{array}{cc}
P_{1} & -P_{1} \\
I & I
\end{array}\right]\left[\begin{array}{l}
\mathcal{L} x(b) \\
\mathcal{L} x(a)
\end{array}\right]
$$

and one can show (this property will be very important in the proof of the exponential stability) that even if $u_{2}$ is chosen equal to zero, there exists an $\varepsilon$ such that:

$$
\left\|u_{1}\right\|^{2}+\left\|y_{1}\right\|^{2} \geq \varepsilon\|\mathcal{L} x(b)\|^{2}
$$

Such result can be generalized to any $u_{2}$ corresponding to conditions at point $a$.

\section{B. DNA-bundle model}

The DNA-bundle is represented by the simple springdamper + load + spring-damper system of Figure 4 and thus admits a port Hamiltonian system representation. In Figure $4 k_{1}, k_{2}, f_{1}, f_{2}$ represent the constants of the springs and the viscous dampers respectively, $M$ is the mass of the load, $x_{c 1}, x_{c 2}$ the relative positions depicted in Figure (4). Let us denote with the sub-index b the system rep-

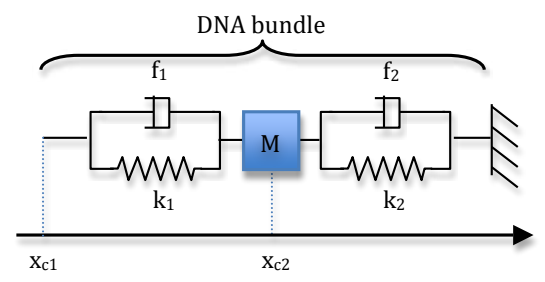

Fig. 4. DNA model

resenting the DNA-bundle. Then we can write by using $v_{b}=\left(x_{c 2}-x_{c 1}, x_{c 2}, M \dot{x}_{c 2}\right)^{T}, u_{b}=\left[\begin{array}{ll}F(b) & T(b)\end{array}\right]^{T}$ and $y_{b}=\left[\begin{array}{ll}v(b) & \omega(b)\end{array}\right]^{T}:$

$$
\left\{\begin{array}{l}
\dot{v}_{b}=\left(J_{b}-R_{b}\right) \frac{d E_{b}}{d v_{b}}+g_{b} u_{b} \\
y_{b}=g_{b}^{T} \frac{d E_{b}}{d v_{b}}+S_{b} u_{b}
\end{array}\right.
$$

with $E_{b}$ the energy of the system (sum of the kinetic and potential energies):

$$
\begin{aligned}
& E_{b}\left(x_{c 2}-x_{c 1},\right.\left.x_{c 2}, M \dot{x}_{c 2}\right)= \\
& \frac{1}{2}\left(k_{1}\left(x_{c 2}-x_{c 1}\right)^{2}+k_{2} x_{c 2}^{2}+M \dot{x}_{c 2}^{2}\right)
\end{aligned}
$$

and

$$
\begin{array}{ccc}
J_{b}=-J_{b}^{T}=\left[\begin{array}{ccc}
0 & 0 & 0 \\
0 & 0 & 1 \\
0 & -1 & 0
\end{array}\right] & R_{b}=R_{b}^{T}=\left[\begin{array}{ccc}
\frac{k_{1}}{f_{1}} & 0 & 0 \\
0 & 0 & 0 \\
0 & 0 & f_{2}
\end{array}\right] \geq 0 \\
g_{b}^{T}=\left[\begin{array}{ccc}
\frac{1}{f_{1}} & 0 & 1 \\
0 & 0 & 0
\end{array}\right] & S_{b}=\left[\begin{array}{cc}
\frac{1}{f_{1}} & 0 \\
0 & \frac{1}{f_{\theta}}
\end{array}\right]>0
\end{array}
$$

where $f_{\theta}$ is the rotational damping constant at the interconnection point. 


\section{THE CONTROLLER+SUSPENSION SYSTEM}

The controller is considered under strict dissipative port Hamiltonian format, i.e. dynamic system of energy $E_{c 3}\left(x_{c 3}\right)=\frac{1}{2} x_{c 3}^{T} Q_{c 3} x_{c 3}$ defined as

$$
\left\{\begin{array}{l}
x_{c 3}=\left(J_{c 3}-R_{c 3}\right) \frac{\partial E_{c 3}\left(x_{c 3}\right)}{\partial x_{c 3}}+B_{c 3} u_{c 3} \\
y_{c 3}=B_{c 3}^{T} \frac{\partial E_{c}\left(x_{c 3}\right)}{\partial x_{c 3}}+D_{c 3} u_{c 3}
\end{array}\right.
$$

with $J_{c 3}=-J_{c 3}^{T}, R_{c 3}=R_{c 3}^{T} \geq 0, D_{c 3}>0$. The suspension system is modelled through a spring/damper system of potential energy:

$$
E_{s}\left(x_{a}\right)=\frac{1}{2} k x_{a}^{2}
$$

The interconnection of these two systems can be written under port Hamiltonian format:

$$
\left\{\begin{array}{l}
\dot{v}_{a}=\left(J_{a}-R_{a}\right) \frac{d E_{a}}{d v_{a}}+g_{a} u_{a} \\
y_{a}=g_{a}^{T} \frac{d E_{a}}{d v_{a}}+S_{a} u_{a}
\end{array}\right.
$$

with $v_{a}=\left(x_{a}, x_{c 3}\right)$ and $E_{a}\left(v_{a}\right)=E_{s}\left(x_{a}\right)+E_{c 3}\left(x_{c 3}\right)$ the energy of the system and:

$$
\begin{aligned}
J_{a}=\left[\begin{array}{cc}
0 & 0 \\
0 & J_{c 3}
\end{array}\right] \quad, R_{a}=\left[\begin{array}{cc}
\frac{1}{D_{c 3}+f} & \frac{B_{c 3}^{T}}{D_{c 3}+f} \\
\frac{B_{c 3}^{T}}{D_{c 3}+f} & R_{c}+\frac{B_{c 3} B_{c 3}^{T}}{D_{c 3}+f}
\end{array}\right] \geq 0 \\
g_{a}=\left[\begin{array}{c}
-\frac{1}{D_{c 3}+f} \\
-\frac{B_{c 3}^{T}}{D_{c 3}+f}
\end{array}\right] \quad S_{a}=\frac{1}{D_{c 3}+f}>0
\end{aligned}
$$

IV. SYSTEMS INTERCONNECTION

In this section we are interested in the interconnection of the infinite dimensional system with the finite dimensional ones. First of all let us gather the two finite dimensional systems as:

$$
\left\{\begin{array}{l}
\dot{v}=\left(J_{c}-R_{c}\right) \frac{\partial E_{c}(v)}{\partial v}+g_{c} u_{c} \\
y_{c}=g_{c}^{T} \frac{\partial E_{c}(v)}{\partial v}+D_{c} u_{c}
\end{array}\right.
$$

with $v=\left(v_{b}, v_{a}\right), u_{c}=\left(u_{b}, u_{a}\right)=(F(b), T(b), F(a)), y_{c}=$ $\left(y_{b}, y_{a}\right)=(v(b), \omega(b), v(a)), E_{c}(v)=E_{b}\left(v_{b}\right)+E_{a}\left(v_{a}\right)$ and

$$
\begin{array}{ll}
J_{c}=-J_{c}^{T}=\left[\begin{array}{cc}
J_{b} & 0 \\
0 & J_{a}
\end{array}\right] & , R_{c}=R_{c}^{T}=\left[\begin{array}{cc}
R_{b} & 0 \\
0 & R_{a}
\end{array}\right] \geq 0 \\
g_{c}=\left[\begin{array}{cc}
g_{b} & 0 \\
0 & g_{a}
\end{array}\right] & S_{c}=\left[\begin{array}{cc}
S_{b} & 0 \\
0 & S_{a}
\end{array}\right]>0
\end{array}
$$

The total energy can be written on the form: $E_{c}(v)=$ $\frac{1}{2} v^{T} Q_{c} v$ with:

$$
Q_{c}=\operatorname{diag}\left(k_{1}, k_{2}, \frac{1}{M}, k, Q_{c 3}\right)>0
$$

The interconnection with the infinite dimensional system is done through the following relation:

$$
\left[\begin{array}{l}
u_{1} \\
y_{1}
\end{array}\right]=\left[\begin{array}{l}
1 \\
0
\end{array}\right] r+\left[\begin{array}{cc}
0 & -1 \\
1 & 0
\end{array}\right]\left[\begin{array}{l}
u_{c} \\
y_{c}
\end{array}\right]
$$

with the additional constraint:

$$
u_{2}=\omega(a)=0
$$

Remark 4.1: From the positivity of $R_{c}$ and the strict positivity of $S_{c}$ the dynamic system defined by (6) is exponentially stable and has all its eigenvalues in the left hand side of the complex plane.

\section{Stability ANALysis of THE ClOSED LOOP SYSTEM}

The exponential stability of the closed loop system is derived following two steps. The first one is concerned with the existence of solutions of the interconneted systems (finite+infinite dimensional systems). It aims at showing that the interconnected system defines a contraction semigroup. The second step consists in finding a Lyapunov function with exponential decay rate. The fact that the closed loop system defines a Boundary Control System with contraction semigroup is derived in Theorem 5.1.

Theorem 5.1: Let the state of the open-loop BCS satisfy $\frac{1}{2} \frac{d}{d t}\|x(t)\|_{\mathcal{L}}^{2}=u(t) y(t)$. Consider the LTI strictly passive finite dimensional system (6) with storage function $E_{c}(t)=$ $\frac{1}{2}\left\langle v(t), Q_{c} v(t)\right\rangle_{\mathbb{R}^{m}}, Q_{c}=Q_{c}^{\top}>0 \in \mathbb{R}^{m} \times \mathbb{R}^{m}$. Then the feedback interconnection (7) of the BCS and the finite dimensional system is again a BCS on the extended state space $\tilde{x} \in \tilde{X}=X \times V$ with inner product $\left\langle\tilde{x}_{1}, \tilde{x}_{2}\right\rangle_{\tilde{X}}=$ $\left\langle x_{1}, x_{2}\right\rangle_{\mathcal{L}}+\left\langle v_{1}, Q_{c} v_{2}\right\rangle_{V}$. Furthermore, the operator $\mathcal{A}_{e}$ defined by

$$
\mathcal{A}_{e} \tilde{x}=\left[\begin{array}{cc}
\mathcal{J} \mathcal{L} & 0 \\
g_{c} \mathcal{C} & J_{c}-R_{c}
\end{array}\right]\left[\begin{array}{l}
x \\
v
\end{array}\right], \quad \mathcal{C} x=I_{c} \tilde{W}\left[\begin{array}{l}
f_{\partial, \mathcal{L} x} \\
e_{\partial, \mathcal{L} x}
\end{array}\right]
$$

with $I_{c}=\left[\begin{array}{ll}I_{3} & 0_{3,1}\end{array}\right]$

$$
D\left(\mathcal{A}_{e}\right)=
$$

$\left\{\left[\begin{array}{l}x \\ v\end{array}\right] \in\left[\begin{array}{l}X \\ V\end{array}\right] \mid \mathcal{L} x \in H^{N}\left(a, b ; \mathbb{R}^{n}\right),\left[\begin{array}{c}f_{\partial, \mathcal{L} x} \\ e_{\partial, \mathcal{L} x} \\ v\end{array}\right] \in \operatorname{ker} \tilde{W}_{D}\right\}$,

where

$$
\tilde{W}_{D}=\left[W+\left[\begin{array}{cc}
S_{c} & 0 \\
0 & 0
\end{array}\right] \tilde{W} \quad\left[\begin{array}{l}
I \\
0
\end{array}\right] g_{c}^{T}\right]
$$

generates a contraction semigroup on $\tilde{X}$.

Proof: The proof is very similar to the one presented in [10, Theorem 5.8, pp:120] and in [16] but we consider here that the feedback is only applied in between $u_{1}$ and $y_{1}$ as stated in (7), $u_{2}$ being freely chosen (equal to zero for the stability analysis). The proof is performed by showing that $\tilde{W}_{D}$ is full rank and by applying the Lumer-Phillips Theorem [12, Theorem 6.1.7, pp:69], which is divided in two parts: showing that $A_{e}$ is a dissipative operator (i.e. $\operatorname{Re}\langle A \tilde{x}, \tilde{x}\rangle \leq$ $0)$ and that $\operatorname{ran}\left(I-A_{e}\right)=\tilde{X}=X \times V$. The fact that $\tilde{W}_{D}$ is full rank is directly derived from the fact that $\left[\begin{array}{l}W \\ \tilde{W}\end{array}\right]$ and $\left[\begin{array}{cccc}I & 0 & S_{c} & 0 \\ 0 & 1 & 0 & 0\end{array}\right]$ ar full rank. From the strict positivity of $D_{c}$ it is easy to show that $A_{e}$ is dissipative using the Kalman-Yakubovich-Popov (KYP) Lemma [19], [20]. The second part of the proof, $\operatorname{ran}\left(I-A_{e}\right)=\tilde{X}$, follows if the matrix $\left(I-\left(J_{c}-R_{c}\right)\right)$ is non-singular. This is true as all the eigenvalues of the matrix $A_{c}$ are in the left half of the complex plane as it is stated in Remark 4.1.

We are now going to prove the exponential stability of the closed loop system by using the aforementioned contraction properties and Lyapunov arguments. The stability results from the strict dissipativity and the exponential stability of the finite dimensional system and the fact that under 
actuation is only considered on the $a$ side of the beam. The Lyapunov candidate that we propose to use is the total energy:

$$
\tilde{E}=E+E_{c}
$$

To prove the result we first need some technical Lemma (the proofs are omitted here but can be found in [13] as they not depend on the fact that the system is partially actuated). These Lemma are concerned with the energy of the finite dimensional system (6) and come from its strict dissipativity and exponential stability properties. Lemma 5.2, 5.3 and 5.4 give bounds on $E_{c}(\tau)$ and $\int_{0}^{\tau} E_{c}(t) d t$ with respect to $\left\|u_{c}(t)\right\|^{2}$ and $\left\|y_{c}(t)\right\|^{2}$.

Lemma 5.2: [13] The energy of (6) satisfies for a positive time $\tau$ :

$$
E_{c}(\tau) \leq \kappa_{1} E_{c}(0)+\kappa_{3} \int_{0}^{\tau}\left\|u_{c}(t)\right\|^{2} d t
$$

where $\kappa_{1}=\kappa_{4} e^{-\kappa_{2} \tau}$ with $\kappa_{2}, \kappa_{3}$ and $\kappa_{4}$ constants.

Lemma 5.3: [13] There exists positive constants $\xi_{1}, \xi_{2}$ and $\tau_{0}$ such for all $\tau>\tau_{0}$ the energy of (6) satisfies

$$
\begin{gathered}
\int_{0}^{\tau} E_{c}(t) d t \leq \xi_{1} \int_{0}^{\tau} v^{\top}(t) Q_{c} R_{c} Q_{c} v(t) d t+ \\
\xi_{2} \int_{0}^{\tau}\left\|u_{c}(t)\right\|^{2} d t
\end{gathered}
$$

Lemma 5.4: [13] For every $\delta_{1}>0$ there exists a $\delta_{2}>0$ such that for all $\tau>0$ the energy of (6) satisfies the relation

$$
\int_{0}^{\tau} \delta_{1} E_{c}(t)+\left\|y_{c}(t)\right\|^{2} d t \leq \delta_{2} \int_{0}^{\tau} E_{c}(t)+\left\|u_{c}(t)\right\|^{2} d t .
$$

We are now going to give some bounds on the total energy of the system in order to prove its exponential stability. For that we mainly need the property (4) i.e:

$$
\left\|u_{1}\right\|^{2}+\left\|y_{1}\right\|^{2} \geq \varepsilon\|\mathcal{L} x(b)\|^{2}
$$

It leads to Lemma 5.5 .

Lemma 5.5: Consider a BCS as described in Theorem 5.1 with $r(t)=0, u_{2}=0$, for all $t \geq 0$. Then, the energy of the system $\tilde{E}(t)=\frac{1}{2}\|x(t)\|_{\mathcal{L}}^{2}+\frac{1}{2} v(t)^{T} Q_{c} v(t)$ satisfies for $\tau$ large enough

$$
\begin{aligned}
& \tilde{E}(\tau) \leq c(\tau) \int_{0}^{\tau}\|(\mathcal{L} x)(t, b)\|_{\mathbb{R}}^{2} d t+\frac{2 c(\tau)}{c_{1}} \int_{0}^{\tau} E_{c}(t) d t \\
& \tilde{E}(\tau) \leq c(\tau) \int_{0}^{\tau}\|(\mathcal{L} x)(t, a)\|_{\mathbb{R}}^{2} d t+\frac{2 c(\tau)}{c_{1}} \int_{0}^{\tau} E_{c}(t) d t
\end{aligned}
$$

where $c$ is a positive constant that only depends on $\tau$ and $c_{1}$ a positive constant.

Proof: The proof is based on a multiplier technic and follows the proof of [13][11]. It mainly uses the contraction property of the semigroup and (4).

These Lemma allow to prove the exponential stability of the closed loop system as stated in Theorem 5.6

Theorem 5.6: Consider the BCS defined by Theorem 5.1 with $r(t)=0, u_{2}=0$, for all $t \geq 0$. Then the finite dimensional system (6) exponentially stabilizes the BCS. by

Proof: The time derivative of the total energy is given

$$
\begin{aligned}
\dot{\tilde{E}}= & -v^{\top} Q_{c} R_{c} Q_{c} v-u_{c}^{\top} S_{c} u_{c} \\
\leq & -v^{\top} Q_{c} R_{c} Q_{c} v-\sigma u_{c}^{\top} u_{c}, \quad \text { since } S_{c} \geq \sigma I \\
= & -v^{\top} Q_{c} R_{c} Q_{c} v-\sigma \epsilon_{1} u_{c}^{\top} u_{c}-\sigma \epsilon_{2} u_{c}^{\top} u_{c} \\
= & -v^{\top} Q_{c} R_{c} Q_{c} v-\sigma \epsilon_{1}\left\|u_{c}\right\|^{2}-\sigma \epsilon_{2}\left\|y_{1}\right\|^{2} \\
= & -v^{\top} Q_{c} R_{c} Q_{c} v-\sigma \epsilon_{1}\left\|u_{c}\right\|^{2}-\sigma \epsilon_{2}\left(\left\|y_{1}\right\|^{2}+\left\|u_{1}\right\|^{2}\right) \\
& +\sigma \epsilon_{2}\left\|u_{1}\right\|^{2}
\end{aligned}
$$

with $\epsilon_{1}+\epsilon_{2}=1$ and where we have used that $u_{c}=-y_{1}$. Using (4)we have

$$
\begin{aligned}
\dot{\tilde{E}} \leq- & v^{\top} Q_{c} R_{c} Q_{c} v \\
& -\sigma \epsilon_{1}\left\|u_{c}\right\|^{2}-\sigma \epsilon_{2} \epsilon\|\mathcal{L} x(t, b)\|^{2}+\sigma \epsilon_{2}\left\|y_{c}\right\|^{2} .
\end{aligned}
$$

Integrating this equation on $t \in[0, \tau]$ we have

$$
\begin{aligned}
& \tilde{E}(\tau)-\tilde{E}(0) \leq-\int_{0}^{\tau} v^{\top}(t) Q_{c} R_{c} Q_{c} v(t) d t+ \\
& -\int_{0}^{\tau} \sigma \epsilon_{1}\left\|u_{c}(t)\right\|^{2}+\sigma \epsilon_{2} \epsilon\|\mathcal{L} x(t, b)\|^{2}-\sigma \epsilon_{2}\left\|y_{c}(t)\right\|^{2} d t .
\end{aligned}
$$

Next choose $\tau$ sufficiently large such that Lemmas 5.3 and 5.5 hold. Using the latter lemma we have

$$
\begin{gathered}
\tilde{E}(\tau)-\tilde{E}(0) \leq-\int_{0}^{\tau} v^{\top} Q_{c} R_{c} Q_{c} v+\sigma \epsilon_{1}\left\|u_{c}\right\|^{2} d t \\
+\frac{\sigma \epsilon_{2} \epsilon}{c(\tau)}\left(\frac{2 c(\tau)}{c_{1}} \int_{0}^{\tau} E_{c}(t) d t-\tilde{E}(\tau)\right)+\sigma \epsilon_{2} \int_{0}^{\tau}\left\|y_{c}\right\|^{2} d t .
\end{gathered}
$$

Grouping terms we have that

$$
\begin{aligned}
& \tilde{E}(\tau)\left(1+\frac{\sigma \epsilon_{2} \epsilon}{c(\tau)}\right)-\tilde{E}(0) \leq \\
& \quad-\int_{0}^{\tau} v(t)^{\top} Q_{c} R_{c} Q_{c} v(t) d t-\sigma \epsilon_{1} \int_{0}^{\tau}\left\|u_{c}(t)\right\|^{2} d t \\
& \quad+\sigma \epsilon_{2}\left(\int_{0}^{\tau} \frac{2 \epsilon}{c_{1}} E_{c}(t)+\left\|y_{c}(t)\right\|^{2} d t\right) .
\end{aligned}
$$

Using Lemma 5.4 with $\delta_{1}=\frac{2 \epsilon}{c_{1}}$ we have

$$
\begin{aligned}
& \quad \tilde{E}(\tau)\left(1+\frac{\sigma \epsilon_{2} \epsilon}{c(\tau)}\right)-\tilde{E}(0) \leq-\int_{0}^{\tau} v(t)^{\top} Q_{c} R_{c} Q_{c} v(t) d t \\
& +\sigma \epsilon_{2} \delta_{2} \int_{0}^{\tau} E_{c}(t) d t+\sigma\left(\epsilon_{2} \delta_{2}-\epsilon_{1}\right) \int_{0}^{\tau}\left\|u_{c}(t)\right\|^{2} d t . \quad
\end{aligned}
$$

Now, using Lemma 5.3 we obtain

$$
\begin{aligned}
& \tilde{E}(\tau)\left(1+\frac{\sigma \epsilon_{2} \epsilon}{c(\tau)}\right)-\tilde{E}(0) \leq \\
& \left(\sigma \epsilon_{2} \delta_{2} \xi_{1}-1\right) \int_{0}^{\tau} v(t)^{\top} Q_{c} R_{c} Q_{c} v(t) d t+ \\
& \sigma\left(\epsilon_{2} \delta_{2}\left(1+\xi_{2}\right)-\epsilon_{1}\right) \int_{0}^{\tau}\left\|u_{c}(t)\right\|^{2} d t .
\end{aligned}
$$

Since $\epsilon_{2}$ may be chosen to be arbitrarily small, i.e, $\epsilon_{2} \ll 1$ and since $\epsilon_{1}=1-\epsilon_{2}$, we finally have that

$$
\tilde{E}(\tau) \leq c_{2} \tilde{E}(0)
$$


with $c_{2}=\frac{1}{\left(1+\frac{\sigma \epsilon_{2} \epsilon}{c(\tau)}\right)}<1$ which proves the theorem.

Remark 5.7: An undamped Timoshenko beam has been considered for the infinite dimensional part of the system. It should be noticed however that the stability proof also holds for a damped Timoshenko beam. Indeed, dissipation in the infinite dimensional model makes the inequalities in Lemma 5.5 strict inequalities.

\section{CONCLUSION}

The model of a DNA-manipulation process has been formulated as a BCpHS. This has been achieved by modelling the sub-systems of the process as port-Hamiltonian systems and showing that the physically meaningful interconnection of the sub-models defines a BCpHS. More specifically, the flexible arm of the micro-gripper has been modelled as a flexible beam (undamped Timoshenko beam) that is clamped on one side, the DNA-bundle as a mass-spring-damper system and the suspension/actuator mechanism as a massspring system. In this model the flexible arm corresponds to a infinite dimensional system, so the complete DNA manipulation processes is the interconnection of an infinite dimensional system and two finite dimensional systems, where one of the input of the infinite dimensional system being assigned to zero. The stabilization has been achieved by interconnecting the suspension/actuator mechanism with a port-Hamiltonian controller (with realistic sensing and actuation variables). The complete process then remains a $\mathrm{BCpHS}$ but with respect a new energy function due to the contribution of the controller. We considered in this paper that the controller has been designed using a dissipative port Hamiltonian format. It has been assumed that no natural damping is present on the infinite dimensional part of the process, which makes the stability result more interesting, since natural damping (which is physically present) adds robustness to the closed-loop system.

Future work aims to include non-linear phenomena in the suspension/actuator mechanism and DNA-bundle.

\section{REFERENCES}

[1] F. Amblard, B. Yurke, A. Pargellis, and S. Leibler, "A magnetic manipulator for studying local rheology and micromechanical properties of biological systems," Review of Scientific Instruments, vol. 67, no. 3 , pp. 818-827, 1996.

[2] C. Gosse and V. Croquette, "Magnetic tweezers: micromanipulation and force measurement at the molecular level," Biophysical Journal, vol. 82, no. 6, pp. 3314-3329, 2002.

[3] R. Simmons, J. Finer, S. Chu, and J. Spudich, "Quantitative measurements of force and displacement using an optical trap," Biophysical Journal, vol. 70, no. 4, pp. 1813-1822, 1996.

[4] E. Florin, V. Moy, and H. Gaub, "Adhesion forces between individual ligand-receptor pairs," Science, vol. 264, no. 5157, p. 415, 1994.

[5] A. Ishijima, T. Doi, K. Sakurada, and T. Yanagida, "Sub-piconewton force fluctuations of actomyosin in vitro," Nature, 1991.

[6] P. Cluzel, A. Lebrun, C. Heller, R. Lavery, J. Viovy, D. Chatenay, and F. Caron, "Dna: an extensible molecule," Science, vol. 271, no. 5250, p. $792,1996$.

[7] C. Bustamante, Z. Bryant, and S. Smith, "Ten years of tension: singlemolecule dna mechanics," Nature, vol. 421, no. 6921, pp. 423-427, 2003.

[8] A. van der Schaft and B. Maschke, "Hamiltonian formulation of distributed-parameter systems with boundary energy flow," Journal of Geometry and Physics, vol. 42, no. 12, pp. 166 - 194, 2002.
[9] Y. Le Gorrec, H. Zwart, and B. Maschke, "Dirac structures and boundary control systems associated with skew-symmetric differential operators,' SIAM Journal on Control and Optimization, vol. 44, no. 5, pp. 1864-1892, 2005.

[10] J. A. Villegas, "A port-Hamiltonian approach to distributed parameter systems," Ph.D. dissertation, Universiteit Twente, 2007.

[11] J. Villegas, H. Zwart, Y. Le Gorrec, and B. Maschke, "Exponential stability of a class of boundary control systems," IEEE Transactions on Automatic Control, vol. 54, pp. 142-147, 2009.

[12] B. Jacob and H. Zwart, Linear Port-Hamiltonian Systems on Infinitedimensional Spaces, ser. Operator Theory: Advances and Applications. Basel, Switzerland: Birkhäuser, 2012, vol. 223.

[13] H. Ramirez, H. Zwart, and Y. Le Gorrec, "Exponential stability of boundary controlled port hamiltonian systems with dynamic feedback," Submitted to the 1st IFAC Workshop on Control of Systems Governed by Partial Differential Equations, Paris, Sept, 25-27, 2013.

[14] A. Macchelli and C. Melchiorri, "Modeling and control of the Timoshenko beam. the distributed port Hamiltonian approach," SIAM Journal on Control and Optimization, vol. 43, no. 2, pp. 743-767, 2004.

[15] A. Macchelli, "Boundary energy shaping of linear distributed portHamiltonian systems," in Proceedings of the 4th IFAC workshop on Lagrangian and Hamiltonian methods for non-linear control, Bertinoro, Italy, August 2012.

[16] H. Ramirez and Y. Le Gorrec, "Exponential stability of a class of PDE's with dynamic boundary control," in Proceedings of the 2013 American Control Conference, Washington, USA, June 2013.

[17] _ - "Boundary port hamiltonian control of a class of nanotweezers," in Proceedings of the European Control Conference 2013, Zurich, Switzerland, July 2013.

[18] M. Boudaoud, Y. Haddab, and Y. Le Gorrec, "Modeling and optimal force control of a nonlinear electrostatic microgripper," Mechatronics, IEEE/ASME Transactions on, vol. PP, no. 99, pp. $1-10,2012$.

[19] J. Willems, "Dissipative dynamical systems part I: General theory," Archive for Rational Mechanics and Analysis, vol. 45, pp. 321-351, 1972.

[20] B. Brogliato, R. Lozano, B. Maschke, and O. Egeland, Dissipative Systems Analysis and Control, 2nd ed., ser. Communications and Control Engineering Series. London: Springer Verlag, 2007. 